Phasing beams with different dispersions and application to the Petawatt-class beamline at the National Ignition Facility

D. Homoelle, J. K. Crane, M. Shverdin, C. L. Haefner, C. W. Siders

September 8, 2010

Applied Optics 
This document was prepared as an account of work sponsored by an agency of the United States government. Neither the United States government nor Lawrence Livermore National Security, LLC, nor any of their employees makes any warranty, expressed or implied, or assumes any legal liability or responsibility for the accuracy, completeness, or usefulness of any information, apparatus, product, or process disclosed, or represents that its use would not infringe privately owned rights. Reference herein to any specific commercial product, process, or service by trade name, trademark, manufacturer, or otherwise does not necessarily constitute or imply its endorsement, recommendation, or favoring by the United States government or Lawrence Livermore National Security, LLC. The views and opinions of authors expressed herein do not necessarily state or reflect those of the United States government or Lawrence Livermore National Security, LLC, and shall not be used for advertising or product endorsement purposes. 


\title{
Phasing beams with different dispersions and application to the Petawatt-class beamline at the National Ignition Facility
}

\author{
D. Homoelle, ${ }^{*}$ J. K. Crane, M. Shverdin, C. L. Haefner, C. W. Siders. \\ Lawrence Livermore National Laboratory, 7000 East Avenue, Livermore, CA 94550, USA \\ *Corresponding author: homoelle1@llnl.gov
}

In order to achieve the highest intensities possible with the short-pulse Advanced Radiographic Capability beamline at the National Ignition Facility (NIF), it will be necessary to phase the individual ARC apertures. This is made especially challenging because the design of ARC results in two laser beams with different dispersions sharing the same NIF aperture. The extent to which two beams with different dispersions can be phased with each other has been an open question. This paper presents results of an analysis that show that the different dispersion values that will be encountered by the shared-aperture beams will not preclude the phasing of the two beams. We also highlight a situation in which dispersion mismatch will prevent good phasing between aperture, and discuss the limits to which higher-order dispersion values may differ before the beams begin to de-phase. (c) 2010 Optical Society of America

OCIS codes: 050.0050, 030.1640, 140.3290, 140.3298, 140.7090.

\section{Introduction}

In order to maximize energy output of a laser system and avoid material damage thresholds and nonlinear propagation effects, it has become common to combine multiple laser outputs in a single aperture [1]- [4]. This can be done either through coherent beam combining, in which all the individual elements operate at the same wavelength, or through wavelength beam combining in which each element operates at a distinct wavelength. In coherent beam combining the relative phase between separate beams must be controlled. While this is challenging for single-wavelength laser systems, it becomes even more challenging when one wishes to coherently combine two short-pulse laser beams with a few nanometers of spectral bandwidth, each of which has a different dispersion. This paper addresses this particular issue 
and how it might affect future experiments that will make use of the Advanced Radiographic Capability (ARC) beamlines at the National Ignition Facility (NIF).

The ARC is one of the many diagnostics being built to support the National Ignition Campaign (NIC) on the NIF [5] and will provide the short-pulse (1-50ps) capability on NIF. Its primary purpose is to provide a sufficient number of energetic x-rays in the range of 10-100 kEV for back-lighting NIF targets [6]- [9]. The ARC system uses four of the 192 NIF beamlines, and each ARC aperture will operate in a split-beam architecture. This splitbeam design is necessitated by both the space restrictions placed on the ARC footprint by the NIF facility and by the fact that the current state-of-the-art diffraction gratings are limited in size to $\sim 1 \mathrm{~m}$ square. In order to avoid cross-talk between neighboring gratings in the compressor, the two beams which share the same ARC aperture experience different dispersions through the laser system [10]. The near-field profile of the NIF-ARC quad is shown in Fig. 1. The individual apertures are rotated because of the spherical geometry of the NIF target chamber. For the purpose of NIC diagnostics, each of the eight ARC beams will be pointed at different targets and staggered in time. This will allow for the generation of an eight-frame back-lit movie of the target capsule implosion.

Simultaneously firing the full ARC quad will give the highest peak intensities on target and will enable many high-energy-density (HED) experiments at the NIF. In order to maximize the laser intensity on target it is desirable that all eight ARC beams be phased with each other. Two other short-pulse, high-energy laser systems, the OMEGA EP Laser System at the Laboratory for Laser Energetics [11] and the Petawatt Aquitaine Laser (PETAL) [12] plan to phase multiple gratings to achieve a large-aperture compressor and a correspondingly tighter focal spot and higher on-target intensity than could be done with a single-gratingsized aperture. This option, however, is not available for the ARC beamline because of the restrictions discussed above. Phasing of the ARC beams requires overcoming many different obstacles that other systems do not always face: the individual beams must be pointed accurately and must have minimal residual spatial phase, and to achieve temporal phasing the path difference between the beams through the NIF laser must be measured to within

a fraction of a wavelength, modulo $2 \pi$. One system has been proposed to achieve all these goals for a single NIF aperture [13]. However, this still leaves open the question of whether the two halves of a single aperture can be phased with each other if they experience different dispersions through the laser system. This paper seeks to address this last concern.

\section{Analysis}

Because we care about how two beams of different dispersions coherently add, we must do the calculations with reference to the electric field and not the intensity, which is a timeaveraged quantity. The electric field in time may be expressed as the real part of the Fourier 
transform of the electric field in frequency space,

$$
E(t)=\Re\left[\mathfrak{F}^{-1}[\underline{E}(\omega)]\right]=\Re\left[\int_{-\infty}^{\infty} \underline{E}(\omega) \exp (i \omega t) \mathrm{d} \omega\right]
$$

where the underbar (e.g $\underline{E})$ indicates a complex quantity. The electric field can be decomposed into a real amplitude and complex phase term

$$
\underline{E}(\omega)=\mathcal{A}(\omega) \exp [i \phi(\omega)]
$$

For a Gaussian spectral amplitude

$$
\mathcal{A}(\omega)=\exp \left[\frac{1}{2}\left(\frac{\omega-\omega_{0}}{\Delta \omega}\right)^{2}\right]
$$

where $\Delta \omega$ is the $1 / e$ width of the spectral intensity.

The spectral phase $\phi(\omega)$ is typically expanded in a Taylor series about the central frequency $\omega_{0}$ and expressed as [14]

$$
\phi(\omega)=\phi\left(\omega_{0}\right)+\phi^{\prime}\left(\omega_{0}\right)\left(\omega-\omega_{0}\right)+\frac{1}{2} \phi^{\prime \prime}\left(\omega_{0}\right)\left(\omega-\omega_{0}\right)^{2}+\frac{1}{6} \phi^{\prime \prime \prime}\left(\omega_{0}\right)\left(\omega-\omega_{0}\right)^{3} \ldots
$$

where $\phi^{\prime}$ indicates a derivative with respect to $\omega$. There is no analytic solution to the integral in Eq. 1 with a phase function given by Eq. 4 so, in general, numerical techniques must be used to calculate $E(t)$. We substitute $\Omega \equiv \omega-\omega_{0}$ into Eqs. 1 and 4 which allows us to factor out the rapidly varying component and results gives us

$$
\begin{aligned}
\int_{-\infty}^{\infty} \underline{E}(\omega) \exp (i \omega t) \mathrm{d} \omega & =\exp \left(i \omega_{0} t\right) \int_{-\infty}^{\infty} \mathcal{A}(\Omega) \exp [i \phi(\Omega)] \exp (i \Omega t) \mathrm{d} \Omega \\
& =\exp \left(i \omega_{0} t\right) \mathfrak{F}^{-1}[\underline{E}(\Omega)]
\end{aligned}
$$

where

$$
\underline{E}(\Omega) \equiv \mathcal{A}(\Omega) \exp [i \phi(\Omega)]
$$

Because the high-frequency oscillations are contained entirely in this factored term, this step will allow us to take a discrete Fourier transform (DFT) of the electric field without excessively fine spectral sampling. This equation allows us to calculate the temporal phase imparted to an individual pulse from a given stretcher/compressor configuration using expressions like those found in Ref. [14].

We can now look at the combined electric field from two beams with different dispersions, which we will refer to as beams $A$ and $B$ :

$$
\begin{aligned}
& \mathfrak{F}^{-1}\left\{\underline{E_{A}}(\Omega)\right\} \equiv \underline{\mathcal{E}_{A}}(t)=\left|\underline{\mathcal{E}_{A}}(t)\right| \exp \left[i \phi_{A}(t)\right] \\
& \mathfrak{F}^{-1}\left\{\underline{E_{B}}(\Omega)\right\} \equiv \underline{\mathcal{E}_{B}}(t)=\left|\underline{\mathcal{E}_{B}}(t)\right| \exp \left[i \phi_{B}(t)\right]
\end{aligned}
$$


Using Eqs. 1, 5, and 7 and factoring back in the rapidly varying component, the electric field in time becomes

$$
\begin{aligned}
& E(t)=\Re\left\{\exp \left[i \omega_{0} t\right] \cdot\left[\mathfrak{F}^{-1}\left\{\underline{E_{A}}(\Omega)\right\}+\mathfrak{F}^{-1}\left\{\underline{E_{B}}(\Omega)\right\}\right]\right\} \\
& E(t)=\Re\left\{\underline{\mathcal{E}_{A}}(t)\left|\exp \left[i \phi_{A}(t)+i \omega_{0} t\right]+\right| \underline{\mathcal{E}_{B}}(t) \mid \exp \left[i \phi_{B}(t)+i \omega_{0} t\right]\right\}
\end{aligned}
$$

and the intensity of the combined beam is

$$
I(t) \sim\langle E(t)\rangle^{2}=\frac{1}{2} \underline{E}(t) \cdot \underline{E}(t)^{*}
$$

where $\underline{E}(t)$ is the complex expression within the $\mathfrak{R}\{\ldots\}$ in Eq. 8 and $\langle\ldots\rangle$ indicates the average over time [16]. Notice that at this stage Eq. 8 is an exact expression for the electric field in time for the combined beams and that $\underline{\mathcal{E}_{A}}(t)$ and $\underline{\mathcal{E}_{B}}(t)$ can be calculated numericallyto any desired precision using Eq. 7 with a DFT.

We can express $\left|\underline{E_{B}}(t)\right|=\left|\underline{E_{A}}(t)\right|+\underline{\delta}(t)$ and then rewrite Eq. 8 as

$$
E(t)=\left|\underline{\mathcal{E}_{A}}(t)\right| \Re\left\{\exp \left(i\left[\phi_{A}(t)+\omega_{0} t\right]\right)+\left(1+\frac{\underline{\delta}(t)}{\left|\underline{\mathcal{E}_{A}}\right|}\right) \exp \left(i\left[\phi_{B}(t)+\omega_{0} t\right]\right)\right\}
$$

If $\underline{\delta}(t) /\left|\underline{\mathcal{E}_{A}}\right| \ll 1$ (i.e. the amplitudes of the two fields are approximately equal and the pulses are of approximately the same width) we can drop the contribution of this term to Eq. 10. In this limit

$$
\begin{aligned}
E(t) & \approx\left|\underline{\mathcal{E}}_{A}(t)\right| \Re\left\{\exp \left(i\left[\phi_{A}(t)+\omega_{0} t\right]\right)+\exp \left(i\left[\phi_{B}(t)+\omega_{0} t\right]\right)\right\} \\
& =\left|\underline{\mathcal{E}_{A}}(t)\right|\left\{\cos \left[\phi_{A}(t)+\omega_{0} t\right]+\cos \left[\phi_{B}(t)+\omega_{0} t\right]\right\} \\
& =2\left|\underline{\mathcal{E}_{A}}(t)\right| \cos \left[\frac{\phi_{A}(t)}{2}-\frac{\phi_{B}(t)}{2}\right] \cos \left[\frac{\phi_{A}(t)}{2}+\frac{\phi_{B}(t)}{2}+\omega_{0} t\right]
\end{aligned}
$$

The slowly varying envelope that describes the temporal beating between beams $A$ and $B$ is contained in the first cosine term, and the rapidly varying part of the electric field is contained in the second cosine term. Thus, in the approximation that $\left|\underline{\mathcal{E}_{B}}(t)\right| \approx\left|\underline{\mathcal{E}_{A}}(t)\right|$ we can see there will be destructive interference when $\cos \left[\phi_{A}(t) / 2-\phi_{B}(t) / 2\right]=0$, or when

$$
\phi_{A}(t)-\phi_{B}(t)=(1 \pm 2 m) \pi, \quad m=0,1,2 \ldots
$$

See the appendix for a discussion of the finer points of calculating the phase using a DFT.

In the ARC geometry, the $A$ and $B$ beams will not be collinear, but side-by-side. So the effect of de-phasing between the two halves will cause those parts of the beam that are out of phase to have a larger focal spot than the parts of the beam that are in phase. This effect is shown in Fig. 2. Figure 2(a) shows the ideal near-field image of a single ARC aperture, and (b)-(d) shows the effect of de-phasing on the far-field spot. The accumulated fluence in the far-field from a split-aperture beam will be a weighted sum of these types of far-field spots. 
Let us now turn our attention back to the expression for the spectral phase $\phi(\omega)$ in Eq. 4 . The $\phi\left(\omega_{0}\right)$ term is simply a constant piston and, for our purposes, we can assume the $A$ and $B$ beams have the same piston. If $A$ and $B$ come from two different front-end laser amplifiers, this would require the output of these amplifiers to be phase-locked to each other. The second

term, $\phi^{\prime}\left(\omega_{0}\right)$ is the time delay of the central frequency through the system [15]. Again, this term can be equalized between the $A$ and $B$ beams by adjusting the total path length in each beamline, and will thus cancel each other in Eq. 12. This leaves only the difference in higherorder dispersion terms that will result in the de-phasing of the $A$ and $B$ electric fields. These terms are known as group delay dispersion (GDD, $\phi^{\prime \prime}\left(\omega_{0}\right)$ ), third-order dispersion (TOD, $\left.\phi^{\prime \prime \prime}\left(\omega_{0}\right)\right)$, and fourth-order dispersion (FOD, $\left.\phi^{\prime \prime \prime \prime}\left(\omega_{0}\right)\right)$.

\section{ARC Dispersion}

We will now apply these results to the range of parameters we might expect to encounter on the ARC beamline. A diagram of the dispersive elements in the ARC beamline is shown in Fig. 3. This paper describes an older, simpler ARC dispersion management design than the one that will be used on NIF. The newer design includes two additional dispersive elements that lead to greater balance in the higher-order dispersion terms and will be described in a future article [18]. We chose to describe the older design in this paper because it is both conceptually simpler and gives worst-case higher-order dispersion values. While the specific values of the older configuration are used in our examples, the result is generally true for any system with similar design. In both cases the ARC aperture has a common short-pulse frontend fiber laser, dubbed the master oscillator (MOR). This beam is split in two, and each half is stretched through separate chirped fiber Bragg gratings (CFBG's) and the dispersion of each of these beams is then fine-tuned with separate pulse-width controllers (PWC's). The pulses then travel through a common amplifier chain in the NIF during which the beams accumulate a moderate amount of material dispersion. Finally, the pulses propagate through the ARC compressor chamber, which has a separate 4-grating compressor for each of the $A$ and $B$ beams, and are then focused onto the target. Because the MOR, the CFBG's, the material, and the compressor gratings all apply fixed dispersions, only the PWC's can be used to adjust the dispersions of the two beams. The PWC's are used to set the pulse-width of each beam. The PWC design parameters are defined in Fig. 4 and the values are given in Table 1. The various contributions to the fixed beamline dispersion for the two beams are given in Table 2. If only the residual GDD component of the the dispersion is included and if a pulse is Gaussian in time, the full-width half-maximum (FWHM) pulsewidth $\tau_{G D D}$ of a non-transform-limited pulse is related to the FWHM width $\tau_{T L}$ of a transform-limited pulse by

$$
\tau_{G D D}=\frac{\sqrt{\tau_{T L}^{4}+(4 \cdot G D D \cdot \log 2)^{2}}}{\tau_{T L}} .
$$


A plot of the pulse width $\tau_{G D D}$ as a function of the PWC separation $L$ for beam $A$ is shown in Fig. 5.

\section{Results}

We will now compute the combined intensities for the $A$ and $B$ beams using Eq. 9. For these calculations we use Gaussian pulses with a spectral intensity FWHM bandwidth of $2.2 \mathrm{~nm}$ centered at wavelength $\lambda_{0}=1053 \mathrm{~nm}$ and a supergaussian filter with 5-nm bandwidth that corresponds to the bandwidth of the compressor. The results of these intensity calculations for three different pulse widths are shown in Fig. 6. Plots of the slowly varying cosine term in Eq. 11 and the phase difference given in Eq. 12 are also given in Figs. 6(a)-(c). The individual intensities for the $A$ and $B$ beams are given in Figs. 6(d)-(f) on the same scale as the combined intensity. These results show that in all cases of interest for the ARC beam the two halves of the beam are substantially in-phase over the length of the pulse despite the difference in residual dispersion experienced by each beam. This can be seen by looking at the values for $\cos \left(\phi_{A} / 2-\phi_{B} / 2\right)$ from Eq. 11(right axis, dotted curve) and $\phi_{A}-\phi_{B}$ (left axis, dashed curve), or by noticing that the peak intensity of the combined beam is $\sim 4$ times the intensity of the individual beams. The structure that is evident in the plots for

the 5-ps pulse is a result of non-zero third-order dispersion values and disappears when the expected lower residual values for the newer ARC dispersion management system are used. This structure is mostly washed out by the time the beams are stretched to $10 \mathrm{ps}$.

A relevant question for system design is how much error is allowable when aligning the compressors and PWC's before the difference in dispersion values results in significant modulation of the combined beam. Because most of the energy is at the peak of the pulse, a large phase difference that occurs at at the peak will cause a significant portion of the beam's energy to be out of phase. We can ask under what condition is the phase difference $\Delta \phi_{A B}(t=0)$ between $A$ and $B$ the closest to an odd multiple of $\pi$ (see Eq. 12). To simplify matters, we will look at the case in which only GDD is present. In this case, there exists an analytic expression for the phase difference at $t=0$ (the peak of a Gaussian pulse) between two pulses with different GDD values:

$$
\Delta \phi_{A B}(t=0)=\frac{1}{2}\left[\operatorname{Arg}\left(-i \mathrm{GDD}_{A}+\frac{\tau^{2}}{4 \log 2}\right)-\operatorname{Arg}\left(-i \mathrm{GDD}_{B}+\frac{\tau^{2}}{4 \log 2}\right)\right]
$$

where $\operatorname{Arg}[\ldots]$ is the argument for the given complex number and $\tau$ is the transform limited FWHM of the intensity. By inspection of Fig. 8 it is clear that the greatest possible value for $\Delta \phi_{A B}$ at $t=0$ is $\pi / 2$. It is also clear from inspection that this will occur when the two GDD values are opposite in sign and much larger than $\tau^{2}$. This can be achieved experimentally by setting the PWC distance $L$ for beam $B$ (or $A$, but not both) to be greater than the value for minimum pulse width, rather than shorter as has been done in the calculation thus 
far (see Table 1), to increase the pulse width. This will cause the GDD for $B$ to have the same magnitude but opposite sign of beam $A$. For this case, Eq. 14 can be evaluated with $\tau=740$ and the values for $\mathrm{GDD}_{A}$ and $\mathrm{GDD}_{B}$ taken from Table 2 except with the sign of $\mathrm{GDD}_{B}$ reversed. For this particular situation, the sign of the TOD is also happens to be reversed, but the sign of the FOD remains the same and the magnitudes of the TOD and FOD remain approximately the same. Equation 14 predicts a phase difference of $\sim 1.497$, and the result calculated using the exact expression in Eq. 9 is shown in Fig. 7 and gives $\Delta \phi_{A B}(t=0)=1.491$, which agrees quite well with the analytic result. Note that the two beams are not only out of phase at $t=0$, but also at several other values across the pulse. Also notice that the approximation used in deriving Eq. 11 is valid in this situation: no approximations were made in calculating the intensity field in Fig. 7a but the minima in the intensity still occur where $\cos \left(\phi_{A} / 2-\phi_{B} / 2\right)$ is zero. These results highlight the importance of both beams having the same sign of GDD to eliminate potential beating between in the combined pulse.

It would be desirable to do a similar analysis to determine the extent to which TOD mismatch leads to de-phasing of the two beams. Unfortunately an analytic solution similar to Eq. 14 does not exist when TOD is included. For this reason, we numerically modeled a representative sample of pulses with varying degrees of TOD mismatch and with the GDD and FOD terms equal to zero. These results are shown in Fig. 9. As can be seen, oscillations that result from the presence of TOD in the pulse, as we have already seen in Fig. 6e and similar to those calculated in other papers [17], have a larger effect on beam shape over the full range of expected TOD variation than any de-phasing between the two beams. We can therefore safely say that TOD should be minimized to limit intensity oscillations, but that the small differences in TOD we might expect to see will not significantly contribute to detrimental de-phasing effects between the two beams. A similar conclusion, that the small differences in FOD that might be expected between the two beams will not be a problem, can be reached by a similar analysis.

\section{Conclusions}

The results presented here indicate that dispersion imbalance between the two halves of the ARC beamline will not be the limiting factor in phasing the ARC aperture. This is a somewhat surprising result given the many electric-field oscillations that occur over the duration of the pulselengths analyzed in this paper. For example, in a 10-ps pulse the electric field will go through over 35,000 oscillations, and the phase difference between the two pulses is much less than $\pi$ over this full range. For the actual laser system great care will still need to be taken to ensure that the two beams are phase-locked coming out of the front end amplifiers, and that the piston phase between the two beams only deviates slightly from an 
odd multiple of $\pi$ while propagating through the laser chain. Given the many challenges and restrictions involved in designing a phased-aperture high-power laser, it is good to know that dispersion imbalance will not be a significant one.

\section{Appendix}

If one wishes to plot the phase of the calculated electric field, rather than a difference in phases as we have done in Eq. 12, some care must be taken. One typically uses a fast Fourier transform (FFT) algorithm to take the DFT. If the beam is centered in the array which will be transformed, then upon taking the FFT, a linear phase term must be added to account for offset of the pulse from zero, corresponding to the fact that $\mathfrak{F}\left[f\left(t-t_{0}\right)\right]=\exp \left[-i \omega t_{0}\right] \cdot \mathfrak{F}[f(t)]$. In general, if NDATA is the number of points in the data array, and NFFT is the number of points used to take the FFT (the array can be padded to give better resolution in the transform space), the output of the FFT must be multiplied by a correction array CORR given by:

$$
\operatorname{CORR}[\mathbf{j}]=\exp [\pi i \cdot \mathrm{NDATA} \cdot \mathbf{j} / \mathrm{NFFT}]
$$

where $\mathbf{j}$ is the index of the array. This correction was not necessary for any of the plots in this paper, since this term cancels when the difference is taken between two phases, as in Eq. 12, but we found it useful during the analysis of these effects to plot the temporal phase of a single beam in order to improve our intuition about these effects.

\section{Acknowledgments}

This work was performed under the auspices of the U.S. Department of Energy by Lawrence Livermore National Laboratory under Contract No. DE-AC52-07NA27344. 


\section{References}

1. T. Y. Fan, "Laser beam combining for high-power, highradiance sources," IEEE J. Sel. Top. Quantum Electron. 11, 567577 (2005).

2. S. J. Augst, J. K. Ranka, T. Y. Fan, and A. Sanchez, "Beam combining of ytterbium fiber amplifiers," J. Opt. Soc. Amer. B, 24, 1707, (2007).

3. C. X. Yu, J.E. Kansky, S.E.J. Shaw, D.V. Murphy, C. Higgs, "Coherent beam combining of large number of PM fibres in 2-D fibre array," Elec. Lett., 42, 1024, (2006).

4. M. A. Vorontsov, T. Weyrauch, L. A. Beresnev, G. W. Carhart, L. Liu, K. Aschenbach, "Adaptive array of phase-locked fiber collimators: analysis and experimental demonstration," IEEE J. Sel. Top. Quanutum Electron., 15, 269 (2009).

5. E. I. Moses, R. N. Boyd, B. A. Remington, C. J. Keane, R. Al-Ayat, "The National Ignition Facility: Ushering in a new age for high energy density science," Phys. Plasmas, 16, 041006 (2009).

6. C.P.J. Barty, M. Key, J. Britten, R. Beach, G. Beer, C. Brown, S. Bryan, J. Caird, T. Carlson, J. Crane, J. Dawson, A.C. Erlandson, D. Fittinghoff, M. Hermann, C. Hoaglan, A. Iyer, L. Jones II, I. Jovanovic, A. Komashko, O. Landen, Z. Liao, W. Molander, S. Mitchell, E. Moses, N. Nielsen, H-H. Nguyen, J. Nissen, S. Payne, D. Pennington, L. Risinger, M. Rushford, K. Skulina, M. Spaeth, B. Stuart, G. Tietbohl, B. Wattellier, "An overview of LLNL high-energy short-pulse technology for advanced radiography of laser fusion experiments," J. Nucl. Fusion, 44, S266 (2004).

7. R. Tommasini, H. S. Park, P. Patel, B.Maddox, S. Le Pape, S. P. Hatchett, B. A. Remington, M. H. Key, N.Izumi, M. Tabak, J. A. Koch, O. L. Landen, D. Hey, A. MacKinnon, J. Seely, G. Holland, L. Hudson, and C. Szabo, "Development of Compton radiography using high-Z backlighters produced by ultra-intense lasers," AIP Conf. Proc. 926, 248 (2007).

8. H.-S. Park, B. R. Maddox, E. Giraldez, S. P. Hatchett, L. T. Hudson, N. Izumi, M. H. Key, S. Le Pape, A. J. MacKinnon, A. G. MacPhee, P. K. Patel, T. W. Phillips, B. A. Remington, J. F. Seely, R. Tommasini, R. Town, J. Workman, and E. Brambrink, "Highresolution $1775 \mathrm{keV}$ backlighters for high energy density experiments," Phys. Plasmas 15, 072705 (2008).

9. "Advanced Radiography: Laser-based X-rays and Gamma-rays," https://lasers.llnl.gov/programs/psa/advanced_radiography/.

10. C. Haefner, J. Heebner, J. Dawson, S. Fochs, M. Shverdin, J.K. Crane, V. K. Kanz, J. Halpin, H. Phan, R. Sigurdsson, W. Brewer, J. Britten, G. Brunton, W. Clark, M. J. Messerly, J. D. Nissen, H. Nguyen, B. Shaw, R. Hackel, M. Hermann, G. Tietbohl, C. W. Siders, C. P. J. Barty, "Characterization of the advanced radiographic capability front 
end on NIF," 2009 UFO/HFSW Conf. Proc., (2009).

11. J. Qiao, A. Kalb, M. J. Guardalben, G. King, D. Canning, and J. H. Kelly, "Largeaperture grating tiling by interferometry for petawatt chirped-pulse-amplification systems," Opt. Express , 15, 9562, (2007).

12. N. Blanchot, E. Bar, G. Behar, C. Bellet, D. Bigourd, F. Boubault, C. Chappuis, H. Coïc, C. Damiens-Dupont, O. Flour, O. Hartmann, L. Hilsz, E. Hugonnot, E. Lavastre, J. Luce, E. Mazataud, J. Neauport, S. Noailles, B. Remy, F. Sautarel, M. Sautet, Cand C. Rouyer, "Experimental demonstration of a synthetic aperture compression scheme for multi-Petawatt high-energy lasers," Opt. Express , 18, 10088, (2010).

13. K. L. Baker, D. Homoelle, E. Utternback, E. A. Stappaerts, C. W. Siders, C. P. J. Barty, "Interferometric adaptive optics testbed for laser pointing, wave-front control and phase," Opt. Express , 17, 16696, (2009).

14. See, for example, S. Backus, C. G. Durfee, M. M. Murnane, H. C. Kapteyn, "High power ultrafast lasers," Rev. Sci. Instr. 69 1207, (1998).

15. E. B. Treacy, "Optical Pulse Compression with Diffraction Gratings," IEEE J. Quantum Electron. , 9 454, (1969).

16. See, for example, D. J. Griffiths, Introduction to Electrodynamics, 2nd Ed. (Prentice Hall, 1989), p. 360.

17. See, for example, G. P. Agraway, "Higher-Order Dispersion," in Nonlinear Fiber Optics, 2nd Ed. (Academic 1995), pp. 75-81, and references therein.

18. C. L. Haefner, J. K. Crane, C. W. Siders, et al., Lawrence Livermore National Laboratory, 7000 East Ave. L-470, Livermore, CA 94550, USA are preparing a manuscript called "Dispersion balance system for the Advanced Radiographic Capability." 
Table 1. Pulse-width Controller Parameters

\begin{tabular}{ccccccc}
\hline \hline & $\lambda_{0}$ & lines $/ \mathrm{mm}$ & $\alpha$ & $\beta$ & $L$ & $\tau_{G D D}$ \\
& $\mathrm{~nm}$ & & degrees & degrees & $\mathrm{mm}$ & $\mathrm{ps}$ \\
\hline $\mathbf{A}$ & 1053 & 1779.4 & 64.31 & 76.5706 & $66-121$ & $50-0.741$ \\
B & 1053 & 1780 & 66.17 & 73.5488 & $193.9-275.7$ & $50-0.741$ \\
\hline \hline
\end{tabular}


Table 2. Dispersion parameters for 740-fs, 5-ps, and 10-ps pulses.

\begin{tabular}{lcccccc}
\cline { 2 - 7 } & \multicolumn{2}{c}{ GDD $\left(\mathrm{ps}^{2} / \mathrm{rad}\right)$} & \multicolumn{2}{c}{ TOD $\left(\mathrm{ps}^{3} / \mathrm{rad}\right)$} & \multicolumn{2}{c}{ FOD $\left(\mathrm{ps}^{4} / \mathrm{rad}\right)$} \\
\cline { 2 - 8 } & $\mathrm{A}$ & $\mathrm{B}$ & $\mathrm{A}$ & $\mathrm{B}$ & $\mathrm{A}$ & $\mathrm{B}$ \\
\hline \hline MOR & \multicolumn{2}{c}{-2.145} & \multicolumn{2}{c}{0.224} & & \\
CFBG & \multicolumn{2}{c}{321.405} & \multicolumn{2}{c}{-6.064} & & \\
\hline PWC 10ps & -26.7139 & -42.3632 & 1.5587 & 1.6631 & -0.1514 & -0.1087 \\
PWC 5ps & -28.0738 & -43.6860 & 1.6381 & 1.7150 & -0.1592 & -0.1121 \\
PWC 0.741ps & -29.3852 & -45.0252 & 1.7146 & 1.7676 & -0.1666 & -0.1155 \\
\hline Material & \multicolumn{2}{c}{0.229} & \multicolumn{2}{c}{0.05298} & & \\
Compressor & -290.1 & -274.46 & 4.4366 & 4.0396 & -0.1151 & -0.1008 \\
\hline \hline Total 10ps & 2.6700 & 2.6637 & 0.2083 & -0.0843 & -0.3495 & -0.2925 \\
Total 5ps & 1.3100 & 1.3409 & 0.2877 & -0.0324 & -0.3572 & -0.29659 \\
Total 0.740ps & -0.0014 & 0.0017 & 0.3642 & 0.0202 & -0.3647 & -0.2994 \\
\hline
\end{tabular}




\section{List of Figures}

1 ARC Quad near-field intensity. Homoelle_fig1_arc_quad.eps . . . . . . . 14

2 ARC aperture near-field intensity (a), and far-field intensities when $A$ and $B$ are in phase (b), $\pi / 2$ out of phase (c), and $\pi$ out of phase (d). Far-field intensities have been normalized to the same scale. Homoelle_fig2_ARC_dephasing_figure.eps . . . . . . . . . . . 15

3 The different dispersive elements in the ARC beamline. Homoelle_fig3_ARC_dispersive_elements.eps . . . . . . . . . . . . 16

4 Tweaker design and design parameter definition. Homoelle_fig4_tweaker_fig.eps . . . . . . . . . . . . . . 17

5 Pulse width as a function of PWC separation $L$ for beam $A$.

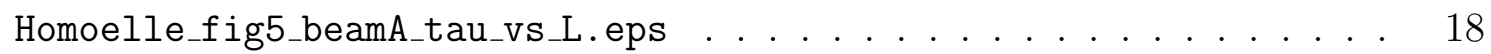

6 Intensity (solid), $\cos \left(\phi_{A} / 2-\phi_{B} / 2\right)$ (dotted, left axis), and $\phi_{A}-\phi_{B}$ (dashed, right axis) for $\sim 740$-fs (a), $\sim 5$-ps (b), and $\sim 10$-ps (c) pulses. The individual intensities for the $A$ and $B$ halves and the combined intensities are plotted in (d)-(f). Homoelle_fig6_all_plots.eps . . . . . . . . . . . . . .

7 Intensity (solid), $\Delta \phi_{A B}$ (dashed, left axis), and $\cos \left(\Delta \phi_{A B} / 2\right)$ (dotted, right axis) (a) and intensities(b) of combined pulse with GDD sign reversed on beam $B$ by adjusting $\mathrm{PWC}_{B}$ distance $L$. Homoelle_fig7_reversed_GDD_phase_int.eps . . . . . . . . . . 20

8 Definition of $\operatorname{Arg}[. .$.$] function used in Eq. 14. Homoelle_fig8_ARG_def .eps .$

9 Intensity profiles for beams with only TOD. $\mathrm{TOD}_{A}=0.364 \mathrm{ps}^{3} / \mathrm{rad}$, and (a) $\mathrm{TOD}_{B}=-0.364$, (b) $\mathrm{TOD}_{B}=-0.121$, and (c) $\mathrm{TOD}_{B}=+0.121 \mathrm{ps}^{3} / \mathrm{rad}$. Homoelle_fig9_TOD_diff_intensities.eps . . . . . . . . . . . 


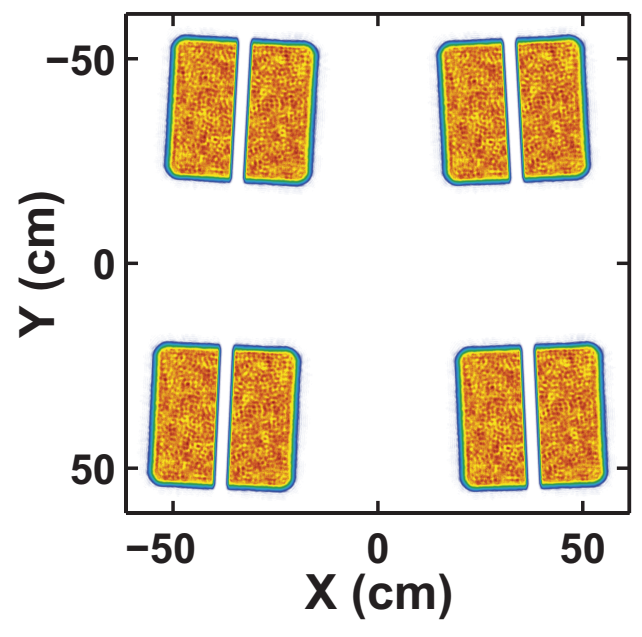

Fig. 1. ARC Quad near-field intensity. Homoelle_fig1_arc_quad.eps 

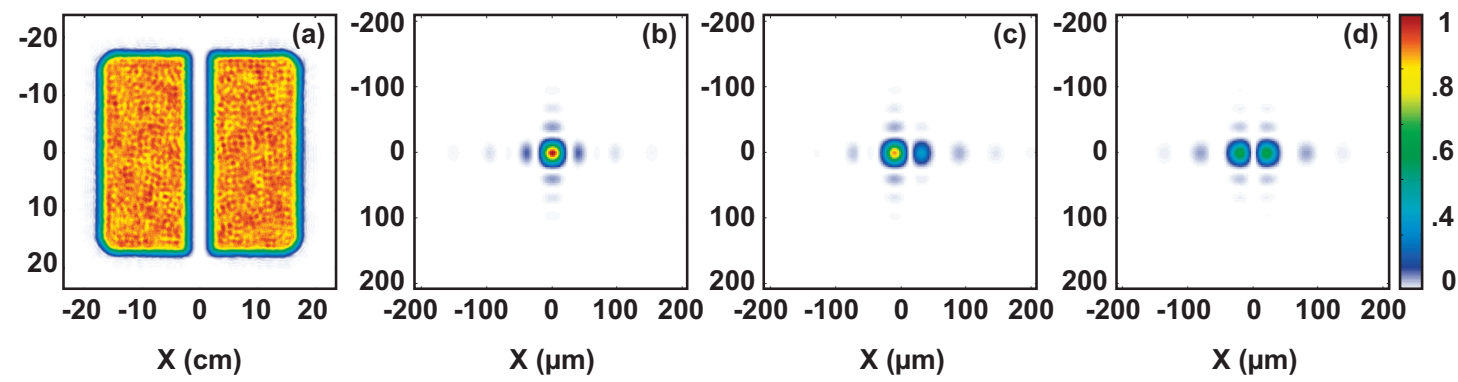

Fig. 2. ARC aperture near-field intensity (a), and far-field intensities when $A$ and $B$ are in phase (b), $\pi / 2$ out of phase (c), and $\pi$ out of phase (d). Far-field intensities have been normalized to the same scale. Homoelle_fig2_ARC_dephasing_figure.eps 


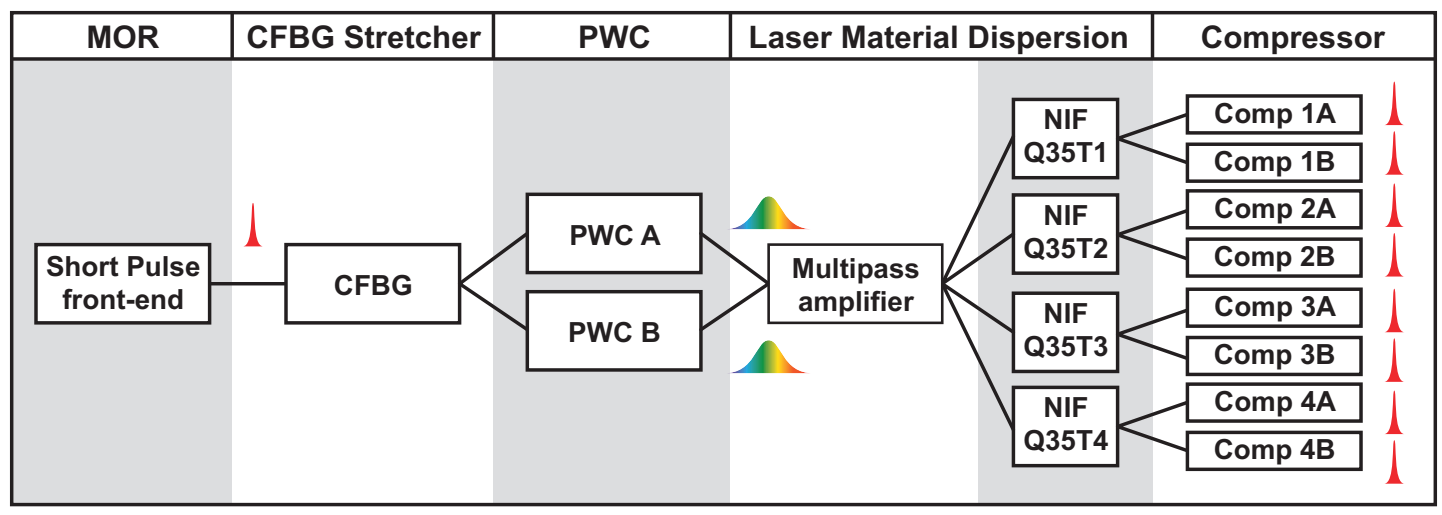

Fig. 3. The different dispersive elements in the ARC beamline. Homoelle_fig3_ARC_dispersive_elements.eps 


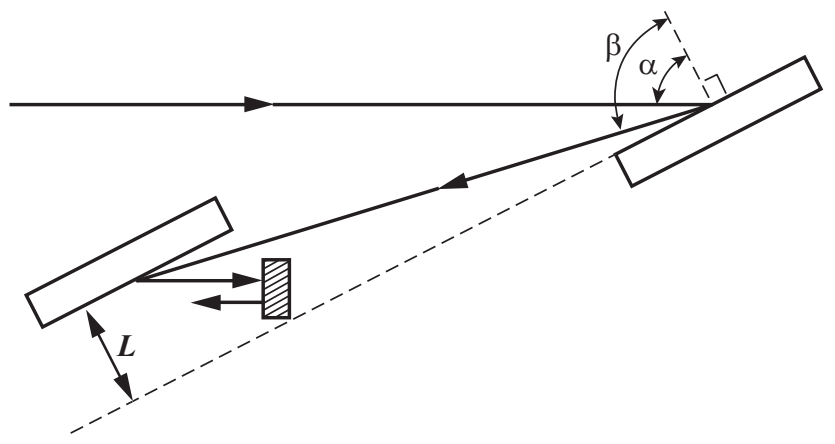

Fig. 4. Tweaker design and design parameter definition. Homoelle_fig4_tweaker_fig.eps 


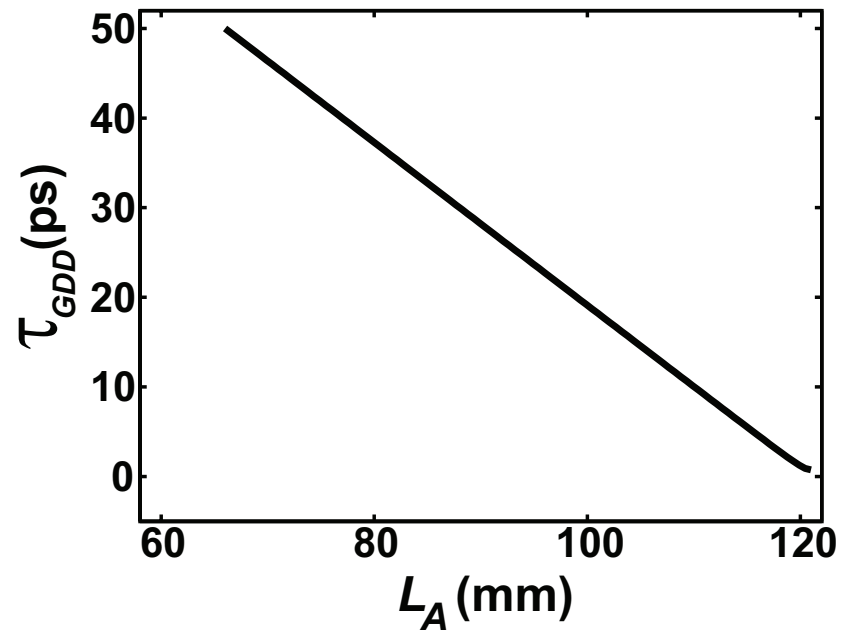

Fig. 5. Pulse width as a function of PWC separation $L$ for beam $A$. Homoelle_fig5_beamA_tau_vs_L.eps 


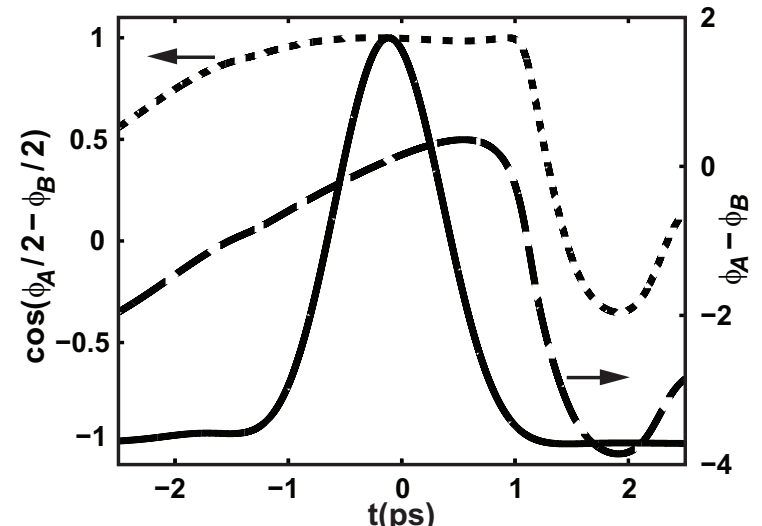

(a)

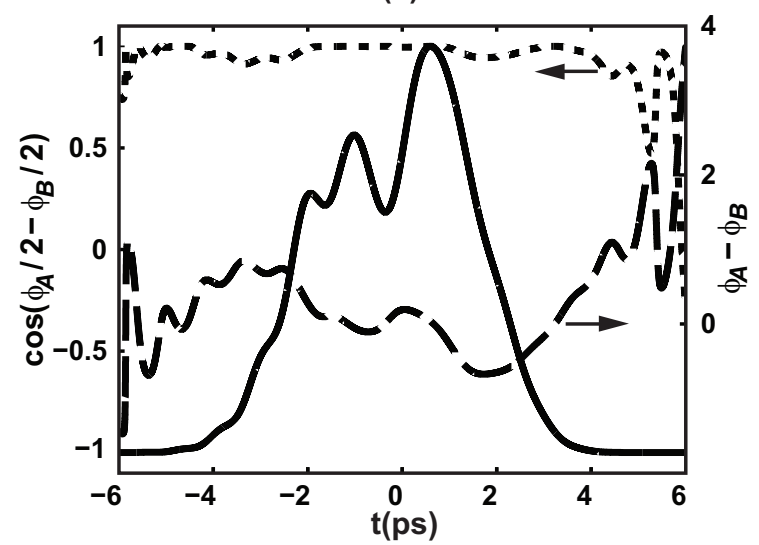

(b)

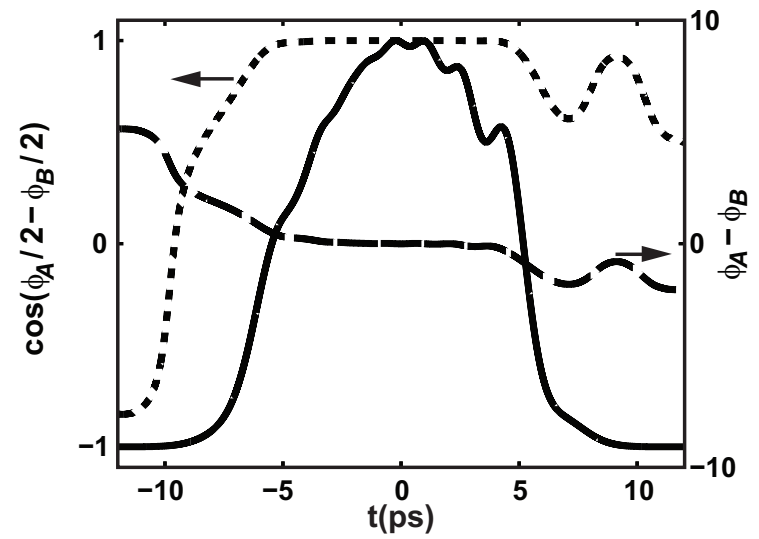

(c)

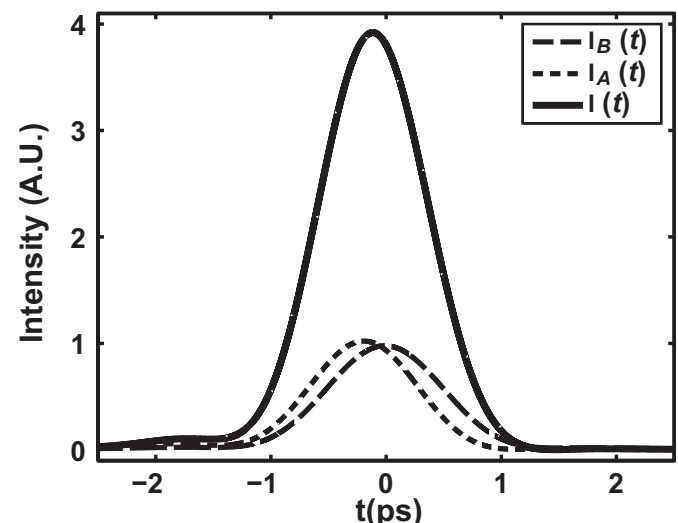

(d)

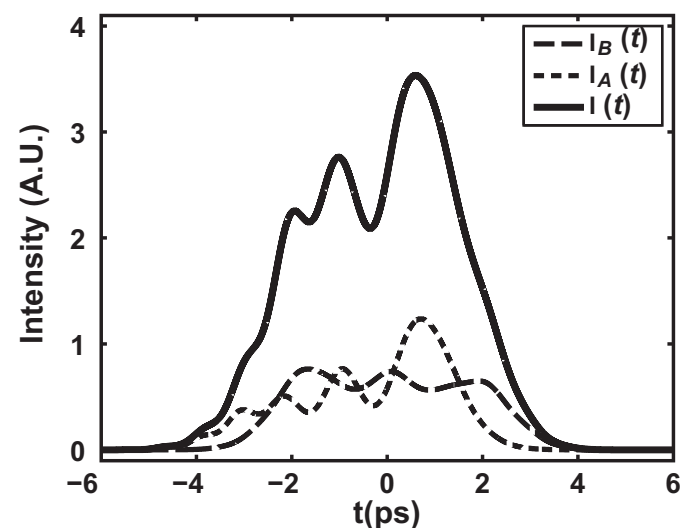

(e)

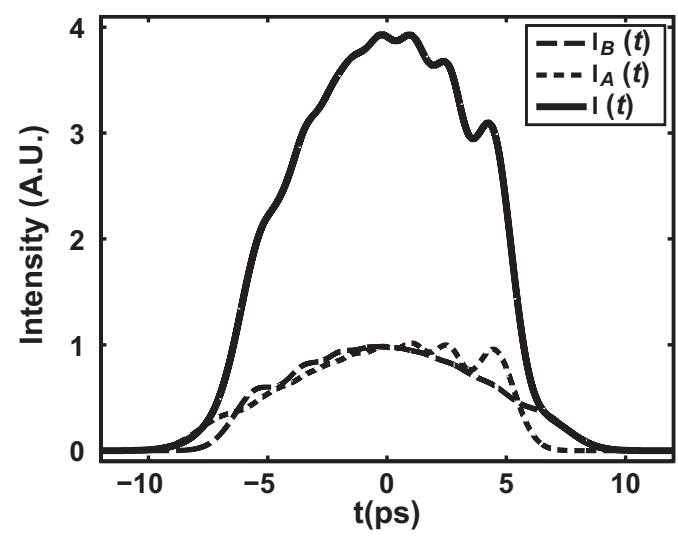

(f)

Fig. 6. Intensity (solid), $\cos \left(\phi_{A} / 2-\phi_{B} / 2\right)$ (dotted, left axis), and $\phi_{A}-\phi_{B}$ (dashed, right axis) for $\sim 740$-fs $(\mathrm{a}), \sim 5$-ps (b), and $\sim 10$-ps (c) pulses. The individual intensities for the $A$ and $B$ halves and the combined intensities are plotted in (d)-(f). Homoelle_fig6_all_plots.eps 


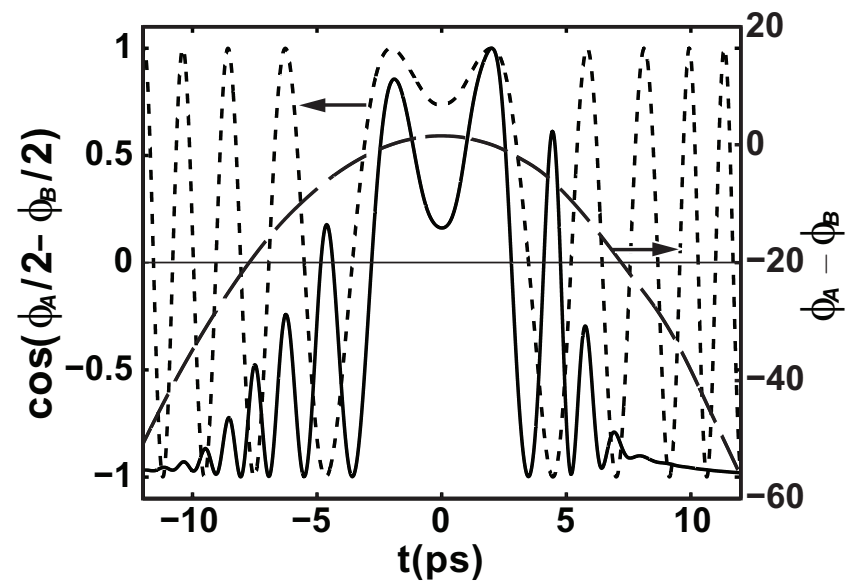

(a)

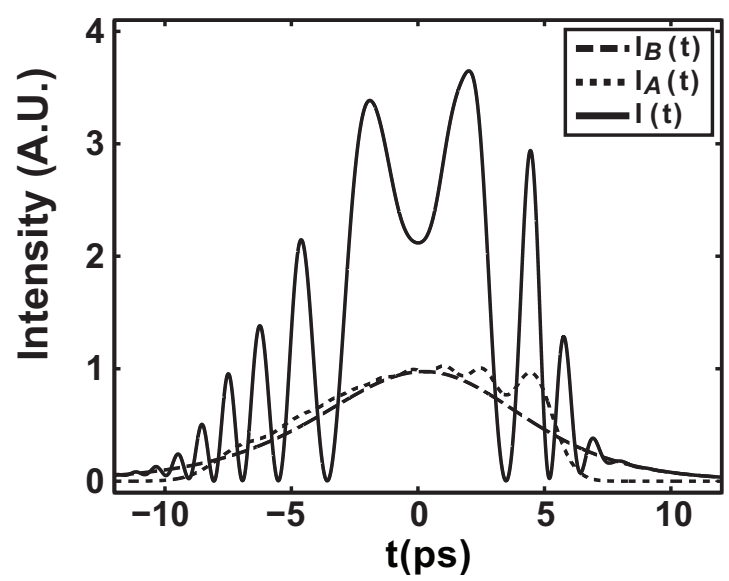

(b)

Fig. 7. Intensity (solid), $\Delta \phi_{A B}$ (dashed, left axis), and $\cos \left(\Delta \phi_{A B} / 2\right.$ ) (dotted, right axis) (a) and intensities(b) of combined pulse with GDD sign reversed on beam $B$ by adjusting $\mathrm{PWC}_{B}$ distance $L$. Homoelle_fig7_reversed_GDD_phase_int.eps 


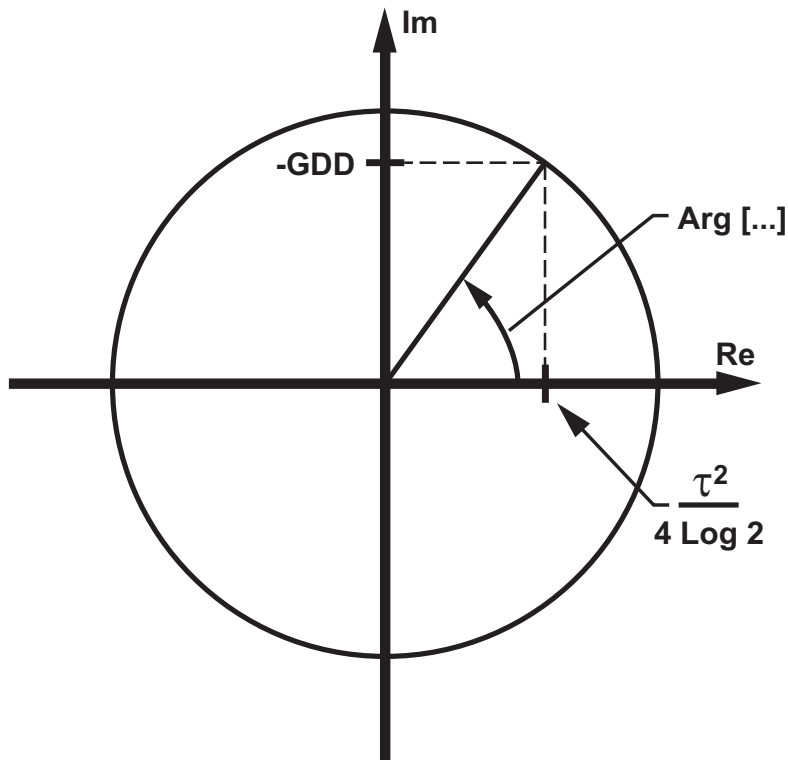

Fig. 8. Definition of $\operatorname{Arg}[\ldots]$ function used in Eq. 14. Homoelle_fig8_ARG_def.eps 


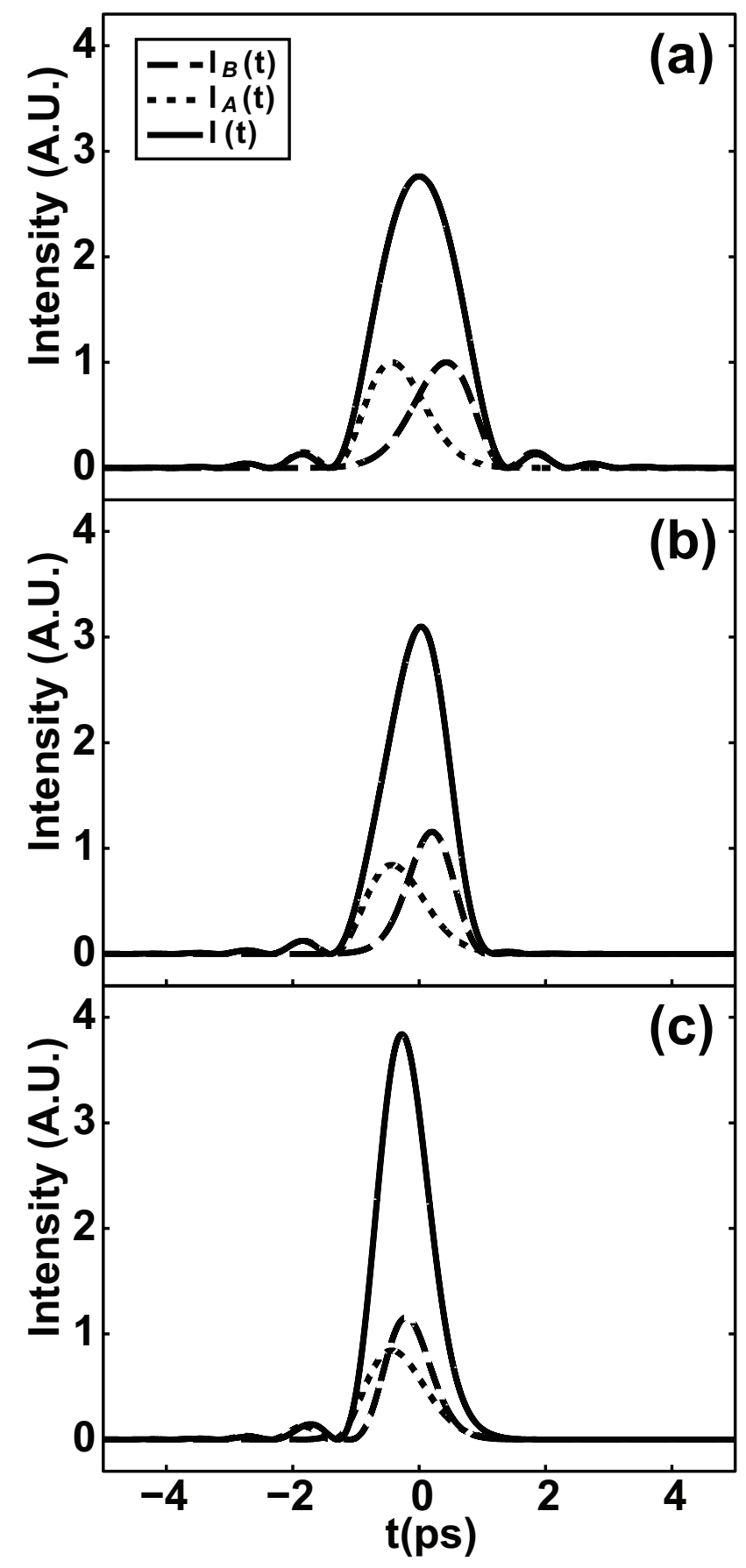

Fig. 9. Intensity profiles for beams with only TOD. $\mathrm{TOD}_{A}=0.364 \mathrm{ps}^{3} / \mathrm{rad}$, and (a) $\mathrm{TOD}_{B}=-0.364$, (b) $\mathrm{TOD}_{B}=-0.121$, and (c) $\mathrm{TOD}_{B}=+0.121 \mathrm{ps}^{3} / \mathrm{rad}$. Homoelle_fig9_TOD_diff_intensities.eps 